\title{
A New Approach to Cutting into Finite Element Models
}

\author{
D. Serby, M. Harders, and G. Székely \\ Swiss Federal Institute of Technology \\ Communication Technology Laboratory \\ ETH Zentrum, CH-8092 Zürich, Switzerland \\ \{dserby, mharders, szekely\}@vision.ee.ethz.ch
}

\begin{abstract}
Virtual reality based surgical simulators offer a very elegant approach to enhancing traditional training in endoscopic surgery. In this context a realistic soft tissue model is of central importance. The most accurate procedures for modeling elastic deformations of tissue use the Finite Element Method to solve the governing mechanical equations. Therapeutic interventions (e.g. cutting) require topological changes of the Finite Element mesh, thus making a non-trivial remeshing step necessary. This paper describes a new approach to cutting in Finite Element models. The central idea is not to introduce new nodes/elements but to displace the existing ones to account for the topological changes introduced by a cut. After the displacement of the nodes/elements the mesh is homogenized to avoid tiny elements which destabilize the explicit time integration necessary for solving the equations of motion.
\end{abstract}

\section{Introduction}

Endoscopic operations have become a very popular technique for the diagnosis and treatment of many kinds of human diseases and injuries. The basic aim of endoscopic surgery is to minimize the damage of the surrounding healthy tissue that is caused by conventional surgery. By employing minimally invasive surgical techniques, the surgeon loses direct contact with the operation site. Performing operations under these conditions demands very specific skills that can be gained only with extensive training. Virtual reality (VR) surgery simulators provide an elegant training possibility.

Within the framework of an earlier project at our institute a realistic, VRbased, endoscopic surgery simulator has been developed [10]. The soft tissue deformations are determined using a complex, non-linear, explicit Finite Element (FE) model. A reduced volume integration scheme (total hourglass control) [5] was applied in order to reduce the computational burden. Furthermore, a parallel computer was built to maintain real-time performance. At present, the simulation is limited to diagnostic interactions. It is, however, desirable to allow also for interactive modifications of the model, especially cutting procedures.

The aim of this paper is to investigate some of the problems that arise while cutting through a FE mesh in a real-time application. We will focus on the 
necessary remeshing caused by the topological changes and present a new cutting algorithm.

\subsection{Previous Work}

Realistic simulation of tissue behaviour during interventions is one of the most challenging tasks in surgical simulations. Several different methods for elastic tissue modeling have been proposed. [11] for instance solves the linear elasticity equations of elastic surface models to calculate deformations.

The two most popular approaches to model soft tissue are physically based mass-spring models and FE models, which are based on continuum mechanics. Whereas the former suffer from poor precision and configuration problems the latter are computationally expensive. There is rich literature on both methods available.

[2] dealt with the interactive simulation of surgical cuts through 3D soft tissue modeled by mass-spring systems. They performed free-form cuts through tetrahedral meshes by applying dynamically adaptive subdivision schemes during the simulation. Any element that is intersected is split into 17 smaller elements once the cutting tool leaves the element, thus dramatically increasing the number of tetrahedra by an average factor of six. 6] has implemented ordinary mass-spring models. In principle using surface models, he adds some additional interior nodes in order to model the volumetric behaviour of the objects. Cutting can only be done along the springs. [4] adopted multi-resolution techniques to optimize the time performance by representing at high resolution only the object parts considered more important. Their approach also allows for topological modifications of tetrahedral mass-spring meshes.

[8] dealt with the simulation of deformable objects using 3D linear FE models. They used various precomputation techniques to achieve real-time performance. Cutting is implemented by removing tetrahedral elements that collide with a virtual scalpel. However, the removal violates the principle of mass conservation. [3] decomposed the anatomical structure into two sections: one onto which they plan to perform cuts and one that will not be affected. Therefore, they propose to apply different elastic models to each section of the anatomy: the more expensive dynamic model is applied only to the section that the user will probably cut. [7] presented a method to cut through soft tissue modeled by a tetrahedral mesh. The tissue deformations are calculated using a linear FE model. The latter can become unstable when the cut is resulting in small elements, which is usually the case. Their examples were not real-time, because they had to run the simulation with a very small integration time step to ensure the stability of the soft tissue model.

After an illustration of the applied FE method in section 2, we will present in section 3 a new cutting algorithm to circumvent some of the problems encountered above. Section 4 describes our results. Finally, we will discuss the improvements and limitations of the new cutting algorithm and future work will be addressed. 


\section{FE Method}

\subsection{Physical Model}

In this section we will describe the model which we use to simulate elastic deformation of an object. Within FE methods, a body is subdivided into a number of finite elements (such as tetrahedra in 3D or triangles in 2D). Displacements and positions in an element are interpolated from discrete nodal values. For every element, the partial differential equations governing the motion of material points of a continuum can be formulated, resulting in the following discrete system of differential equations [1]:

$$
M \ddot{U}+C \dot{U}+K U=R
$$

where $\boldsymbol{U}$ is the vector of nodal displacements, $\boldsymbol{M}$ is the mass matrix, $\boldsymbol{C}$ is the damping matrix, $\boldsymbol{K}$ is the stiffness matrix and $\boldsymbol{R}$ is the vector of external node forces. Given an isotropic solid no time-consuming, numerical integration is necessary to determine the stiffness matrix.

The time integration of the above system of equations can be performed using implicit or explicit integration schemes. For the implicit method, the solution has to be calculated iteratively at every discrete time step. In contrast to this, the explicit time integration can be performed without iteration and without solving a system of linear algebraic equations. This integration scheme is only conditionally stable. The critical integration step $\Delta t_{c r}$ is given by the Courant-FriedrichsLewy condition. It is approximately proportional to the characteristical element length $\Delta L_{\text {min }}$ of the model:

$$
\Delta t_{c r} \approx \frac{\Delta L_{\min }}{c}
$$

where $c$ is the speed of sound in the medium.

In order to calculate a solution for (1) the time-dependent variables must be discretized. We use the explicit Euler central difference terms as estimates of the continuous variables and formulate the equation of motion at the time $t$ :

$$
\begin{aligned}
& \left(\frac{1}{\Delta t^{2}} \boldsymbol{M}+\frac{1}{2 \Delta t} \boldsymbol{C}\right){ }^{t+\Delta t} \boldsymbol{U}= \\
& { }^{t} \boldsymbol{R}-\left(\boldsymbol{K}-\frac{2}{\Delta t^{2}} \boldsymbol{M}\right){ }^{t} \boldsymbol{U}-\left(\frac{1}{\Delta t^{2}} \boldsymbol{M}-\frac{1}{2 \Delta t} \boldsymbol{C}\right){ }^{t-\Delta t} \boldsymbol{U}
\end{aligned}
$$

Assuming lumped masses at the nodes, we can use diagonal damping and mass matrices. In this case, (3) can be easily solved for the unknown nodal displacements at the time $t+\Delta t$.

\subsection{Problems when Cutting in FE Meshes}

Purely diagnostic interactions require only one generation of the FE mesh at the beginning of the simulation. In contrast to this, cutting into a FE mesh results in 
topological changes which demand a remeshing process after each cut. In order to conserve the stability of the integration scheme it is crucial to prevent the element size $\Delta L_{\text {min }}$ from decreasing rapidly during the cutting process as this requires the adequate adjustment of the critical integration step $\Delta t_{c r}$. To this end, an appropriate remeshing has to be performed after each cut.

Another issue, when cutting into a FE mesh, is the dimension of the system matrices. If during the cutting process the number of elements is increasing, the calculation effort to set up the system matrices as well as to solve the corresponding equations of motion increases, too. Again, an appropriate remeshing should prevent this.

In the next section a new cutting algorithm will be introduced. In order to understand the performance as well as the limitations of our concept we performed an initial study in the 2D case. Nevertheless, we always ensured the extensibility of our approach to the third dimension.

\section{Cutting Algorithm}

\subsection{Overview}

In order to avoid a rapid growth of the number of nodes/elements, no new nodes, except for the necessary duplicates on the cut line, are introduced after performing a cut. Instead, the nearest existing nodes will be displaced into the cut points to account for the topological changes caused by a cut. The displacements result in a more or less distorted mesh. Several edges can become quite small, bringing about instabilities when integrating the equations of motion. Therefore, it is necessary to homogenize the mesh in a subsequent step. To this end, a combination of a linear mass-spring and a particle system is applied.

The particle system provokes a relatively homogeneous arrangement of the nodes. However, it does not ensure the preservation of the mesh topology. The mass-spring system is well suited for this task but it fails to completely rectify the mesh. For these reasons, a suitable combination of both systems will be utilized. After this brief presentation of our concept, we will give an in-depth explanation of the necessary steps performed by the cutting algorithm.

\subsection{Detailed Description}

We start from a triangular mesh through which a straight cut is laid (see Fig. 1(a)]. First of all, the cut is divided into a number of segments of equal length. In a next step the number of cut points, which was deliberately chosen too high, has to be adapted to the present mesh. To this end, the distance between each cut point and its nearest neighbour is determined. If any two cut points have the same nearest neighbour, the number of cut points is reduced by one and the new cut points are determined. This process is repeated until no two cut points have the same nearest neighbour (see Fig. 1(b)]. Thereupon, the nearest neighbours are displaced to the cut point positions (see Fig. 2(a). 


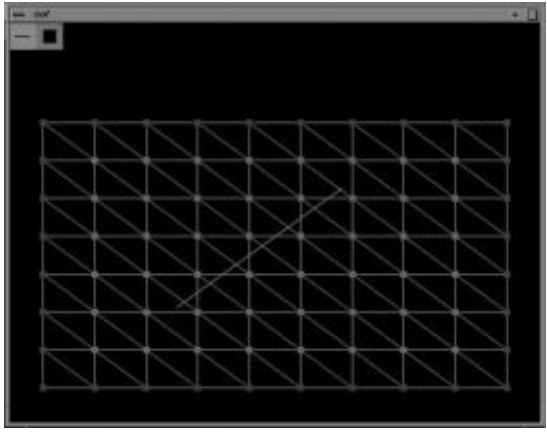

(a) Cut through initial mesh

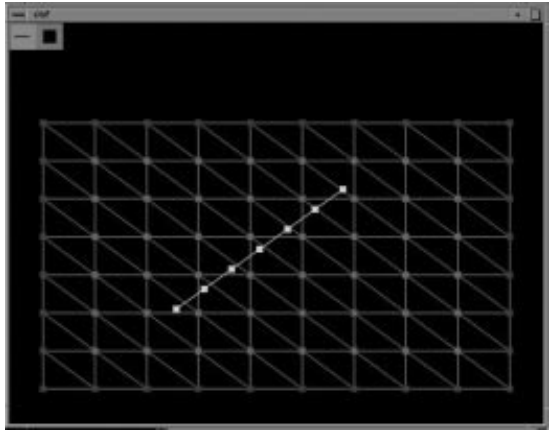

(b) Adaption of number of cut points to current mesh

Fig. 1. First steps of the cutting algorithm

The adaption process prevents the occurrence of all but two mesh errors. First, a cut line segment can sometimes be intersected by another element edge. The second error occurs when all three nodes of an element are displaced into cut points, causing it to degenerate into a line. Both kinds of errors are traced and corrected. In the next step, the combined mass-spring/particle system is applied. Since the mesh distortion occurs mainly in a neighbourhood of the cut, we have limited the homogenization process to the vicinity of the cut to reduce the computational cost. Nodes are considered as mass points resp. particles and element edges as springs. There are two kinds of internal forces that affect the nodes: On the one hand the spring forces $\boldsymbol{g}_{i j}$, on the other hand the forces $\boldsymbol{f}_{i}{ }^{i n t}$ resulting from the potential energy function $\phi$ of the particle system:

$$
\boldsymbol{g}_{i j}=k_{i j}\left(\left\|\boldsymbol{x}_{j}-\boldsymbol{x}_{i}\right\|-l_{i j}\right) \frac{\left(\boldsymbol{x}_{j}-\boldsymbol{x}_{i}\right)}{\left\|\boldsymbol{x}_{j}-\boldsymbol{x}_{i}\right\|}, \quad \boldsymbol{f}_{i}^{i n t}=-\nabla_{\boldsymbol{x}_{i}} \phi
$$

We used the Lennard-Jones potential $\phi_{L J}$ [9] because it creates long-range attractive forces and short range repulsive forces which encourage particles to maintain equal spacing. The Lennard-Jones energy function $\phi_{L J}$ is defined as a function of separation distance $r$ between a pair of particles:

$$
\phi_{L J}(r)=\frac{B}{r^{n}}-\frac{A}{r^{m}}
$$

A single node $i$ of the combined mass-spring/particle system is governed by the following equation of motion:

$$
m_{i} \ddot{\boldsymbol{x}}_{i}+c_{i} \dot{\boldsymbol{x}}_{i}+\left(s_{\text {spring }} \sum_{j} \boldsymbol{g}_{i j}+s_{\text {particle }} \boldsymbol{f}_{i}^{i n t}\right)=\boldsymbol{f}_{i}
$$

where $s_{\text {spring }}$ and $s_{\text {particle }}$ are scaling factors. In order to solve the above dynamic problem the explicit Euler scheme is applied. The new positions of the movable 
nodes are calculated until the displacements drop below a given threshold. Then, additional postprocessing-steps are performed, which optimize the area of the elements. The result of the whole remeshing process is shown in Figure 2(b). Finally, in order to allow the mesh to open a partner node is generated for each cut point. The original node belongs to elements on one side of the cut and the duplicated node belongs to elements on the other side of the cut.

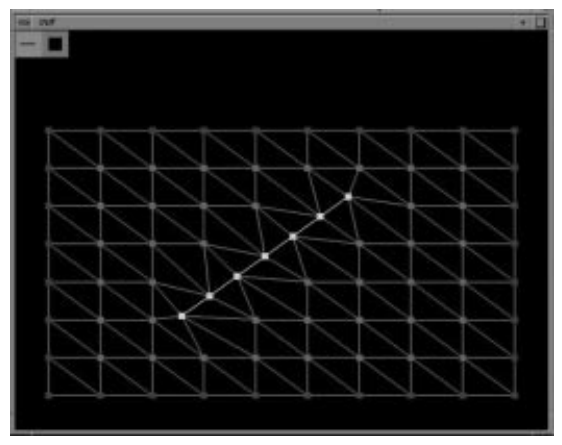

(a) Neighbours dislocated into cut points

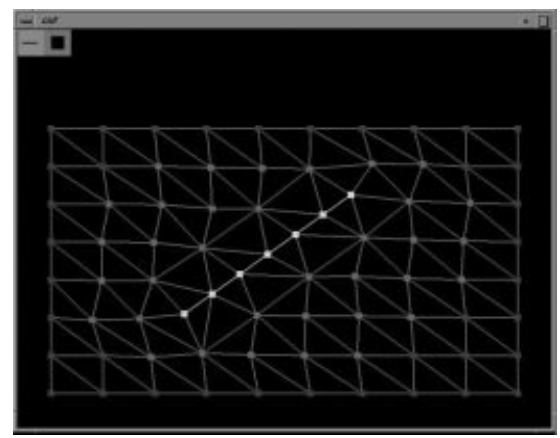

(b) Mesh after homogenization process

Fig. 2. Dislocation and homogenization process

\section{Results and Discussion}

Several different configurations regarding the kind of mesh and the run of the cut were examined. In order to analyze the quality of the remeshing process a number of statistical values were determined: the number of nodes $\# N$, the number of elements \#E, the minimum element area $A_{\min }$ and edge length $E_{\text {min }}$, the maximum element area $A_{\max }$ and edge length $E_{\max }$, the median element area $A_{\text {med }}$ and edge length $E_{\text {med }}$, the standard deviation of the element area $A_{\sigma}$ and of the edge length $E_{\sigma}$ and the computing time $t$. All the statistical values were determined before (I) and after (NR) the cut was performed. In the case of the regularly meshed rectangle, these values were also determined for another kind of remeshing (OR) for comparison purposes. Within the OR, at every intersection of the cut line with the mesh a new node is introduced together with the necessary corresponding elements.

All experiments were carried out on a one-processor SGI Octane $(195 \mathrm{MHz}$; $128 \mathrm{MB}$ RAM). An excerpt of the examples is listed in table1, Figure 3(a) shows as an example a homogeneous mesh through which a cut was laid. In order to do a FE calculation boundary and initial conditions were applied and material and geometric parameters were allocated. Subsequently, the equations of motion 
were solved by means of a commercial FE software. A snapshot of the solution at a certain point in time is shown in Figure $3(\mathrm{~b})$.

Table 1. Statistical values of the example configurations

\begin{tabular}{|c|c|c|c|c|c|c|c|c|c|c|c|c|}
\hline Config. & Mesh & \begin{tabular}{|c}
$\# \mathbf{N}$ \\
{$[-]$}
\end{tabular} & $\begin{array}{c}\mathbf{E} \\
{[-]}\end{array}$ & $\begin{array}{l}\mathbf{A}_{\min } \\
{\left[\mathrm{mm}^{2}\right]}\end{array}$ & $\begin{array}{l}\mathbf{A}_{\max } \\
{\left[\mathrm{mm}^{2}\right]}\end{array}$ & $\begin{array}{l}\mathbf{A}_{\text {med }} \\
{\left[\mathrm{mm}^{2}\right]}\end{array}$ & $\begin{array}{c}\mathbf{A}_{\sigma} \\
{\left[\mathrm{mm}^{2}\right.}\end{array}$ & $\begin{array}{l}\mathbf{E}_{\min } \\
{[\mathrm{mm}]}\end{array}$ & $\begin{array}{l}\mathbf{E}_{\max } \\
{[\mathrm{mm}]}\end{array}$ & $\begin{array}{l}\mathbf{E}_{\text {med }} \\
{[\mathrm{mm}]}\end{array}$ & $\begin{array}{c}\mathbf{E}_{\sigma} \\
{[\mathrm{mm}]}\end{array}$ & $\begin{array}{c}\mathbf{t} \\
{[s]}\end{array}$ \\
\hline \multirow[t]{3}{*}{$\overline{1}$} & I & 80 & $\overline{126}$ & 22.22 & 22.22 & 22.22 & 0.00 & 5.7 & 9.7 & 7.7 & 1.4 & \\
\hline & OR & 106 & 152 & 2.24 & 22.22 & 18.42 & 7.10 & 2.2 & 9.7 & 6.9 & 2.2 & \\
\hline & NR & 85 & 126 & 14.46 & 33.01 & 22.22 & 3.10 & 4.8 & \begin{tabular}{|l|}
10.6 \\
\end{tabular} & 7.7 & 1.5 & 3.09 \\
\hline \multirow[t]{3}{*}{2} & $I$ & 320 & 570 & 4.91 & 4.91 & 4.91 & 0.00 & 2.7 & 4.6 & 3.6 & 0.7 & \\
\hline & OR & 376 & 626 & 0.00 & 4.91 & 4.47 & 1.29 & 0.1 & 4.6 & 3.4 & 1.0 & \\
\hline & NR & 326 & 570 & 3.56 & 7.62 & 4.91 & 0.24 & 2.3 & 4.9 & 3.6 & 0.7 & 23.30 \\
\hline \multirow[t]{2}{*}{3} & I & \begin{tabular}{|l}
76 \\
\end{tabular} & 119 & 26.20 & 26.20 & 26.2 & 0.00 & 7.8 & 7.8 & 7.8 & 0.0 & \\
\hline & NR & 83 & 312 & 13.88 & 39.88 & 26.20 & 4.43 & 4.8 & 11.2 & 7.8 & 0.9 & 2.75 \\
\hline \multirow[t]{2}{*}{4} & I & 312 & 555 & 5.88 & 5.88 & 5.88 & 0.00 & 3.7 & \begin{tabular}{|l}
3.7 \\
\end{tabular} & 3.7 & 0.0 & \\
\hline & NR & 327 & 555 & 2.45 & 8.26 & 5.88 & 0.61 & 2.2 & 4.9 & 3.7 & 0.3 & 35.96 \\
\hline \multirow[t]{2}{*}{5} & I & 45 & 69 & 3.37 & 53.54 & 24.59 & 12.33 & 2.8 & \begin{tabular}{|l|}
13.6 \\
\end{tabular} & 7.6 & 2.3 & \\
\hline & NR & 48 & 69 & 3.37 & 53.54 & 24.59 & 12.48 & 2.8 & 13.6 & 7.7 & 2.3 & 0.77 \\
\hline \multirow[t]{2}{*}{$\overline{6}$} & I & 120 & 200 & 0.83 & 20.87 & 8.82 & 4.78 & 1.2 & 9.9 & 4.6 & 1.6 & \\
\hline & NR & 124 & 200 & 0.83 & 23.84 & 8.82 & 5.00 & 1.2 & 9.2 & 4.6 & 1.6 & 3.05 \\
\hline
\end{tabular}

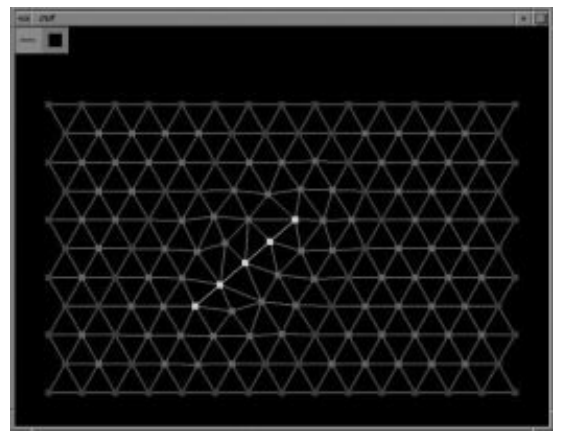

(a) Result of the remeshing with the cutting algorithm

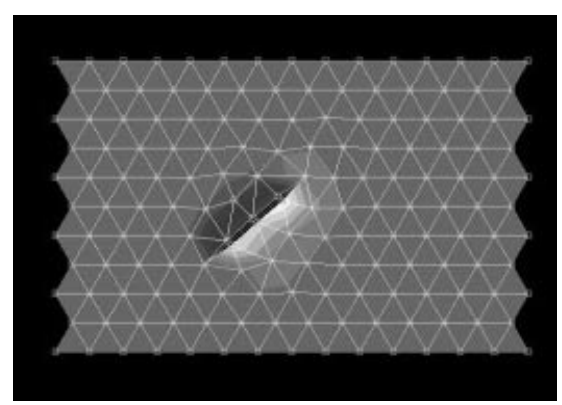

(b) Output of the FE calculation at a certain point in time

Fig. 3. Example showing the result of the remeshing and the FE calculation

The following remarks refer to specific configurations but they are valid in general. Configuration 1 in the table 1 shows that there were 80 nodes and 126 elements before cutting, and 85 nodes and the 126 elements afterwards when using the NR. This compares very favorably to the same cut performed by the OR. While the minimum element area and the minimum edge length are heavily decreasing with the OR, there is only a slight decline with the NR. Configuration 2 points out that the median values and the standard deviations remain approximately the same before and after cutting with the NR. In contrast to this, the 
OR results in considerably different median values and standard deviations. Regarding the computing time, it has to be noted, that there is a disproportionate increase from smaller to larger meshes. This is due to the currently inefficient implementation of the post-processing step, which unnecessarily investigates the whole mesh. Due to the local nature of the homogenization process, it is sufficient to also limit the subsequent optimization to the vicinity of the cut, thus making the running time of this step directly proportional to the number of cut points and not to the mesh size.

\section{Conclusion and Perspectives}

We have presented a new algorithm for cutting into a FE mesh. The technique of dislocating the nodes prevents the number of elements from increasing. The additional nodes that are generated, are the unavoidable partner nodes at the cut points. Therefore, the size of the system matrices stays at a reasonable level, while cutting the mesh. The homogenization procedure restores a more or less homogeneous mesh after the node dislocations. Thus, the problem of decreasing element size is minimized and consequently the stability of the solution of the equations of motion is increased. The computing time necessary for the remeshing takes up only a small fraction of the overall time our surgery simulator needs for the FE calculations and the collision detection, respectively. Due to the fact, that the computing time of our approach is only dependent on the number of cut points, its real-time capability is guaranteed even for larger models.

Future work will focus on analyzing the inter-dependence of the parameters of the combined system (e.g. spring constants) in order to adjust them optimally for a specific mesh, thereby improving the homogenization process. Also the possibility of combining progressive cutting with the current algorithm will be investigated. Finally, we will research further into the problems arising in the 3D case, where whole tetrahedral faces have to be dislocated into the cutting plane.

\section{References}

1. Klaus-Jürgen Bathe. "Finite-Elemente-Methoden". Springer Verlag, Berlin Heidelberg, 1990.

2. Daniel Bielser, Volker. A. Maiwald, and Markus H. Gross. "Interactive Simulation of Surgical Cuts". Proceedings of Pacific Graphics 2000, IEEE Computer Society Press, 2000.

3. Stéphane Cotin, Hervé Delingette, and Nicholas Ayache. "Efficient Linear Elastic Models of Soft Tissues for real-time surgery simulation". Research Report No. 3510, INRIA Sophia Antipolis, 1998.

4. Fabio Ganovelli, Paolo Cignoni, Claudio Montani, and Roberto Scopigno. "A Multiresolution Modell for Soft Objects supporting interactive cuts and lacerations". EUROGRAPHICS 2000, 2000.

5. Roland Hutter, and Peter F. Niederer. "Fast Accurate Finite Element Algorithm for the Calculation of Organ Deformations". ASME Summer Bioengineering Conference, Big Sky, Montana, 1999. 
6. Christian Kuhn. "Modellbildung und Echzeitsimulation deformierbarer Objekte zur Entwicklung einer interaktiven Trainingsumgebung für die Minimal-Invasive Chirurgie". Dissertation, Fakultät für Informatik, Universität Karlsruhe, 1997.

7. Andrew B. Mor and Takeo Kanade. "Progressive Cutting with Minimal New Element Creation". In Proceedings MICCAI 2000, Third International Conference, Pittsburgh, PA, 2000.

8. Morten-Bro Nielsen. "Finite Element Modelling in Surgery Simulation". In Proceedings MMVR 1997, San Diego, CA, 1997.

9. David Love Tonnesen. "Dynamically Coupled Particle Systems for Geometric Modeling, Reconstruction, and Animation". Thesis, Graduate Department of Computer Science, University of Toronto, 1998.

10. G. Székely et al. "Virtual Reality-Based Simulation of Endoscopic Surgery". Presence, Vol. 9, June 2000, 310-333, Massachusetts Insitute of Technology, 2000.

11. Demetri Terzopoulos, John Platt, Alan Barr, and Kurt Fleischer. "Elastically Deformable Models". Computer Graphics, Volume 21, Number 4, July 1987, 1987. 